\title{
Three new branched chain equations of state based on Wertheim's perturbation theory
}

\author{
Bennett D. Marshall and Walter G. Chapman ${ }^{a)}$ \\ Department of Chemical and Biomolecular Engineering, Rice University, 6100 S. Main St. Houston, \\ Texas 77005, USA
}

(Received 18 February 2013; accepted 14 April 2013; published online 3 May 2013)

\begin{abstract}
In this work, we present three new branched chain equations of state (EOS) based on Wertheim's perturbation theory. The first represents a slightly approximate general branched chain solution of Wertheim's second order perturbation theory (TPT2) for athermal hard chains, and the second represents the extension of first order perturbation theory with a dimer reference fluid (TPT1-D) to branched athermal hard chain molecules. Each athermal branched chain EOS was shown to give improved results over their linear counterparts when compared to simulation data for branched chain molecules with the branched TPT1-D EOS being the most accurate. Further, it is shown that the branched TPT1-D EOS can be extended to a Lennard-Jones dimer reference system to obtain an equation of state for branched Lennard-Jones chains. The theory is shown to accurately predict the change in phase diagram and vapor pressure which results from branching as compared to experimental data for $\mathrm{n}$-octane and corresponding branched isomers. () 2013 AIP Publishing LLC. [http://dx.doi.org/10.1063/1.4803023]
\end{abstract}

\section{INTRODUCTION}

Molecular branching can have a significant effect on the phase behavior of short chain molecules such as alkanes ${ }^{1}$ and long chain polymers such as polyethylene. ${ }^{2}$ The general effect of branching is to decrease attraction between molecules due to the fact that the backbone chains can no longer position themselves in mutual configurations which maximizes the Van der Waals forces between molecules. This decrease in effective attraction results in a corresponding decrease in critical temperatures and boiling points. ${ }^{1}$

An accurate approach to modeling chain molecule fluids is the statistical associating fluid theory (SAFT) equation of state. ${ }^{3}$ SAFT is a perturbation theory typically written with a hard sphere reference fluid; the free energy contribution due to chain formation is obtained from Wertheim's first order perturbation theory $\mathrm{TPT}^{4-7}$ and long range attractions can be included using a mean field approximation, ${ }^{8}$ square well potential of variable range, ${ }^{9}$ the PCSAFT approach, ${ }^{10}$ or some other method. The common thread between these approaches is the use of TPT1 for the chain formation contribution to the free energy.

Wertheim's theory was originally developed as a primitive model for hydrogen bonding (associating) fluids. A key feature of any theory to model associating fluids is that the theory must account for the saturation of hydrogen bonds. ${ }^{4,6}$ To accomplish this, Wertheim developed a multi-density approach where each bonding state of the molecule was assigned a density. With this multi-density formalism, Wertheim developed an exact cluster expansion. The theory is typically applied as a perturbation theory which treats association as

\footnotetext{
a) Author to whom correspondence should be addressed. Electronic mail: wgchap@ rice.edu. Tel.: (1) 713.348.4900. Fax: (1) 713.348.5478.
}

a perturbation to a hard sphere reference fluid. ${ }^{7}$ Of course, there are many levels of perturbation, in TPT1 all graphs in the cluster expansion which contain more than a single association bond are neglected, mathematically this means that non-nearest neighbor interactions in associated clusters are neglected. This allows for the development of a very simple equation of state which has proven to be reasonably accurate. ${ }^{11,12}$ Even though only pair interactions are considered complex associated chains and clusters can be formed. Going to second order in perturbation TPT $2^{7}$ all graphs with a single path of three attraction bonds are retained in addition to the graphs retained in TPT1, this form of the theory now includes interactions between bonded pairs and next nearest neighbors along the chain.

Wertheim's theory was extended to mixtures by Chapman. ${ }^{13}$ Chapman ${ }^{13}$ considered a stoichiometric mixture of hard spheres which could be bonded together to form hard chain molecules with a fixed chain length. To irreversibly form the chain, association energies were allowed to become infinitely large. This was carried out in TPT1 by Chapman ${ }^{13}$ resulting in a simple first order chain equation of state which forms the basis of all of the SAFT approaches mentioned previously. Since the chain equation of state is derived in TPT1, it cannot distinguish between various isomers of the same chain length. To include this structural information one must go to a minimum of TPT2, this was a problem initially tackled by Phan et al. ${ }^{14}$ Starting from a stoichiometric mixture of hard spheres Phan et al. ${ }^{14}$ developed an equation of state for hard sphere linear chains in TPT2; further, they were also able to develop a TPT2 equation of state for the chain formation of athermal star molecules. The equation of state for star molecules could not be obtained in closed form and had to be evaluated numerically. The general effect of including branching in the TPT2 equation of state for star molecules was a 
decrease in pressure as compared to the linear isomer. Recently, Marshall and Chapman ${ }^{15}$ extended this approach further by developing a general form for the TPT2 correction to the free energy for any branched molecule in terms of a sum over all triplet partitions of the molecule, unfortunately this sum has not been evaluated at this time.

The theories discussed above are all formulated around a hard sphere monomer reference fluid; however, Wertheim's perturbation theory can also be applied to other reference fluids such as a fluid of dimers which consist of two spheres bonded at contact. This was the approach taken by Ghonasgi and Chapman ${ }^{16}$ and Chang and Sandler ${ }^{17}$ in the development of the TPT1-D (D stands for dimer) equation of state, also known as SAFTD. The extension of the theory from TPT1-M (M stands for monomer reference fluid) to TPT1-D is straightforward with the only additional difficulty being the requirement of the orientationally averaged dimer-dimer end to end pair correlation function. The TPT1-D equation of state has been shown to be superior to either TPT1 or TPT2 with monomer reference fluids for the prediction of hard chain properties for both pure components ${ }^{16}$ and mixtures. ${ }^{18}$ The improved accuracy of TPT1-D over TPT1-M is a result of the fact that more intramolecular information is included in the theory when dimers are used as the building block. Since TPT1-D is still a first order theory, it cannot distinguish between linear and branched isomers of the same chain length.

As mentioned previously, the various hard chain equations of state are paired with a dispersion free energy contribution to obtain a useful equation of state for modeling the phase equilibria of real fluids. An alternative to this approach is to have the attractive interactions built into the reference fluid. This is the approach taken by Chapman ${ }^{19}$ who extended TPT1-M to associating spheres with a Lennard-Jones (LJ) reference system, Ghonasgi and Chapman ${ }^{20}$ who developed a TPT1-M chain equation of state with a LJ reference fluid, and Johnson et al. ${ }^{21}$ who developed a TPT1-M equation of state for LJ chains ${ }^{21}$ which consist of chains of LennardJones spheres bonded at the point $r=\sigma_{L J}$ where $\sigma_{L J}$ is the point where the LJ potential is zero. This approach was later extended to a LJ dimer reference fluid by Johnson ${ }^{22}$ who developed TPT1-D for LJ fluids; shortly thereafter it was shown that the LJ TPT1-D theory gave significantly improved predictions for the phase equilibria of $\mathrm{LJ}$ chains as compared to the LJ TPT1-M monomer reference fluid theory. ${ }^{23}$ TPT2 has not been applied with a LJ reference system due to the lack of availability of a LJ triplet correlation function.

Our goal here is to obtain a simple equation of state for branched molecules which includes the effect of branching in the reference chain equation of state. As mentioned previously, Phan et al. ${ }^{14}$ developed a branched TPT2 theory for star molecules, but this approach requires a numerical solution which is not amiable for combination with a long range attraction contribution to model real systems. In order to obtain an approximate simple analytical form for the TPT2 correction to the free energy for branching Blas and $\mathrm{Vega}^{24}$ used the TPT2 solution for linear chains of Phan et al. ${ }^{14}$ to include the effect of branching. Using this method the actual topology of the molecule was not included and the majority of the molecule was treated in TPT1.
Here, we will take a different approach. We will construct the second order correction to the free energy for branched molecules from the second order free energy of smaller linear chains. It is then shown that the general form for the free energy obtained through TPT2 arguments can also be evaluated using the TPT1-D free energy to develop a TPT1-D theory for branched chains. It is shown that both equations of state for hard chains (athermal chains) give improved predictions for compressibility factors of star molecules and second virial coefficients of dendrimers as compared to their linear counterparts. We also compare the predictions of our approximate TPT2 solution to the numerical TPT2 solution for star molecules of Phan et al. ${ }^{14}$ to show the two give nearly identical results, validating our approximation scheme.

These two branched hard chain equations of state could be used as the basis of a perturbation theory to model the phase behavior of real fluids; however, an alternative method is to use a Lennard-Jones reference fluid. A significant advantage of the TPT1-D version of the branched theory is the ability to extend it to a LJ reference fluid. Using the correlated LJ monomer and dimer correlation functions of Johnson, ${ }^{22}$ the branched TPT1-D approach can be easily extended to a LJ dimer reference system. By comparison to experimental data for the phase diagram and vapor pressure of n-octane and corresponding branched isomers, we show that the new equation of state accurately predicts the effects of branching without introducing any additional complexity to the linear form of the theory.

The paper is organized as follows. In Sec. II, a general TPT2 equation of state is developed for branched hard chain molecules. We then apply the form of the TPT2 solution to extend TPT1-D to account for topology in branched chain molecules. In Sec. III, the branched TPT1-D equation of state is extended to a Lennard-Jones dimer reference system and compared to experimental data for the phase behavior of $n$ octane and corresponding branched isomers.

\section{BRANCHED HARD CHAINS}

In this section, a general branched hard chain equation of state based on TPT2 will be developed. Using the general form of the free energy derived in the framework of TPT2, the TPT1-D equation of state ${ }^{16}$ will be extended to account for branching. First let us define the reduced excess free energy per chain molecule $f_{q}^{E}$ of a fluid in a volume $V$, temperature $T$, and composed of $N_{q}$ chain molecules of length $q$ as

$$
f_{q}^{E}=\frac{A_{q}^{E}}{N_{q} k_{B} T},
$$

where $A_{q}^{E}$ is the excess Helmholtz free energy of a system of chains of length $q$. For the remainder of this paper, the reduced excess free energy per chain molecule of length $q f_{q}^{E}$ will simply be referred to as the "free energy." The free energy is given as the sum of first order free energy $f_{q}^{\text {TPT1 }}$ and the second order correction for triplet connectivity

$$
f_{q}^{E}=f_{q}^{T P T 1}+f_{q}^{T P T 2}
$$


The free energy $f_{q}^{\text {TPT1 }}$ is given as the sum of the reference system excess free energy $f_{q}^{R E F}$ and a contribution for TPT1 chain connectivity at the pair level ${ }^{11}$

$$
f_{q}^{T P T 1}=f_{q}^{R E F}-(q-1) \ln g_{R E F}(\sigma),
$$

where $g_{R E F}(\sigma)$ is the reference system pair correlation function at contact. For a hard sphere reference, we use the Carnahan and Starling result ${ }^{25}$

$$
g_{R E F}(\sigma)=g_{H S}(\sigma)=\frac{1-\eta / 2}{(1-\eta)^{3}},
$$

where $\sigma$ is the hard sphere diameter, the packing fraction $\eta=\frac{\pi}{6} q \rho_{q} \sigma^{3}$, and $\rho_{q}$ is the number density of molecules composed of $q$ spherical segments. For linear chains, the TPT2 correction is given by the solution of Phan et al. ${ }^{14}$

$$
\begin{aligned}
f_{q}^{T P T 2}= & -\ln \left(\frac{1}{\sqrt{1+4 \lambda}}\right) \\
& -\ln \left(\left[\frac{1+\sqrt{1+4 \lambda}}{2}\right]^{q}-\left[\frac{1-\sqrt{1+4 \lambda}}{2}\right]^{q}\right) .
\end{aligned}
$$

For fixed bond angle $\alpha$, the term $\lambda$ is given by ${ }^{7}$

$$
\lambda=\frac{g_{H S}^{(3)}(\sigma, \sigma, 2 \sigma \sin (\alpha / 2))}{g_{H S}(\sigma) g_{H S}(\sigma)}-1 .
$$

For flexible molecules, $\lambda$ is obtained by multiplying Eq. (6) by the step function

$$
\theta(\alpha)= \begin{cases}2 / 3 & \alpha \geq \pi / 3 \\ 0 & \alpha<\pi / 3\end{cases}
$$

and integrating over all bond angles. Here, we will use the result of Phan et al. ${ }^{14}$ for a hard sphere reference system

$$
\begin{aligned}
\lambda & =\int_{0}^{\pi} \theta(\alpha)\left(\frac{g_{H S}^{(3)}(\sigma, \sigma, 2 \sigma \sin (\alpha / 2))}{g_{H S}(\sigma) g_{H S}(\sigma)}-1\right) \sin \alpha d \alpha \\
& \approx 0.2336 \eta+0.1067 \eta^{2} .
\end{aligned}
$$

The term $\lambda$ given by Eq. (8) is small; this results in the term $(1-\sqrt{1+4 \lambda}) /(1+\sqrt{1+4 \lambda})$ also being small which to a very good approximation can be neglected. ${ }^{14}$ Rearranging Eq. (5) and neglecting the small term above, the TPT2 correction for flexible linear chains is now for $q \geq 3$

$$
\begin{aligned}
f_{q}^{T P T 2}= & -\ln \left(\frac{1}{\sqrt{1+4 \lambda}}\right) \\
& -\ln \left(\left[\frac{1+\sqrt{1+4 \lambda}}{2}\right]^{q}\left(1-\left[\frac{1-\sqrt{1+4 \lambda}}{1+\sqrt{1+4 \lambda}}\right]^{q}\right)\right) \\
\approx & -\ln \left(\frac{1}{\sqrt{1+4 \lambda}}\right)-q \ln \left(\frac{1+\sqrt{1+4 \lambda}}{2}\right) .
\end{aligned}
$$

For $q<3$, the TPT2 correction to the free energy is $f_{q}^{\text {TPT2 }}$ $=0$. We note the approximation in Eq. (9) is highly accurate due to the fact that the small term $(1-\sqrt{1+4 \lambda}) /$ $(1+\sqrt{1+4 \lambda})$ is raised to the $q \geq 3$ power which is very small when compared to unity. For this reason, this term can

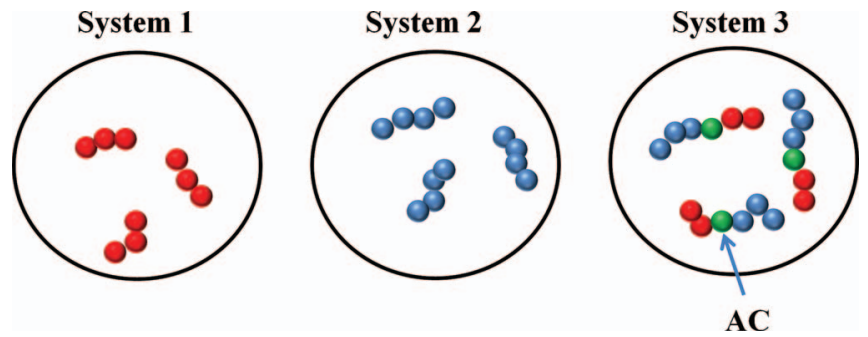

FIG. 1. Three systems composed of chains of length $q=3, s=4$, and $q+s-1=6$.

be safely neglected. An important feature of Eq. (9) is that it is linearly dependant on chain length where Eq. (5) is not. Now we wish to extend this result to branched molecules. The contributions to the free energy for a general molecule composed of $m$ segments $f_{m}^{H S}$ and $f_{m}^{\text {TPT1 }}$ do not depend on the topology of the molecule; however, $f_{m}^{\text {TPT2 }}$ does depend on molecular topology.

To obtain a general branched TPT2 equation of state, we will construct the TPT 2 contribution to the free energy of a branched molecule $f_{m}^{\text {TPT2 }}$ from the free energies for smaller linear chains. What we wish to determine is the difference in free energies between branched and linear isomers. We begin by considering three separate systems each at a temperature $\mathrm{T}$ and packing fraction $\eta$; the first system is a fluid of linear chains of length $q$ of TPT2 free energy $f_{q}^{\text {TPT2 }}$, the second contains chains of length $s$ with TPT2 free energy $f_{s}^{\text {TPT2 }}$, and the third is composed of chains of length $q+s-1$ with free energy $f_{q+s-1}^{\mathrm{TPT} 2}$. An example is given in Fig. 1 for $q=3$ and $s=4$, the segment type labeled AC is thought of as an articulation segment obtained by superimposing two shorter chains of length $q=3$ and $s=4$ to create a two arm star polymer composed of an articulation segment and arms of length 2 and 3 ; this is equivalent to a linear chain of length 6 . The TPT2 correction to the free energy of system 3 can be written as

$$
f_{q+s-1}^{\mathrm{TPT} 2}=f_{q}^{\mathrm{TPT} 2}+f_{s}^{\mathrm{TPT} 2}+\Delta f_{q s}^{\mathrm{TPT} 2},
$$

where $\Delta f_{q s}^{\mathrm{TPT} 2}=f_{q+s-1}^{\mathrm{TPT} 2}-f_{q}^{\mathrm{TPT} 2}-f_{s}^{\mathrm{TPT} 2}$ which can be evaluated with Eq. (9) for $q, s \geq 3$ from which we obtain the result

$$
\Delta f_{q s}^{T P T 2}=\ln \left(\frac{1}{\sqrt{1+4 \lambda}}\right)+\ln \left(\frac{1+\sqrt{1+4 \lambda}}{2}\right) .
$$

Of the utmost importance is the fact that Eq. (11) is independent of the lengths $s$ and $q$ (a fact which will prove useful in developing a branched chain free energy) allowing us to drop the subscripts and simply write $\Delta f_{q s}^{T P T 2}=\Delta f^{T P T 2}$. In the Appendix, it is shown that this is true even when $q, s=2$. This independence of chain lengths results from the linear dependence on chain length in the slightly approximate free energy in Eq. (9).

Following this same logic, now we would like to form a system of chain molecules of chain length $m=q+s+u-2$ with a single branch. We consider four systems each at a packing fraction $\eta$ and temperature $T$ : system 1 is composed of chains of length $q$, system 2 chains of length $s$, system 3 contains chains of length $u$, and system 4 contains branched 


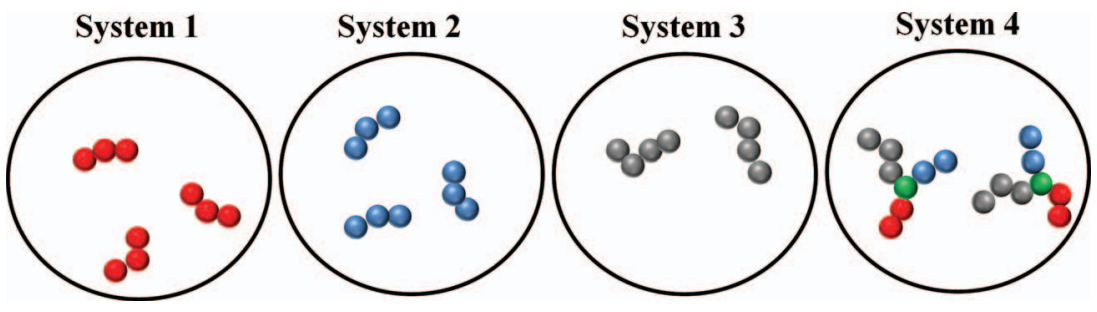

FIG. 2. Four systems with $q=3, s=3$, and $u=4$.

molecules composed of $m=q+s+u-2$ segments. An example of these systems is given in Fig. 2 for $q=3, s=3$, and $u=4$. The TPT 2 corrections to the free energies of the systems can then be written as

$$
f_{m}^{T P T 2}=f_{q}^{T P T 2}+f_{u}^{T P T 2}+f_{s}^{T P T 2}+\Delta f_{q s u}^{T P T 2},
$$

where $f_{m}^{T P T 2}$ is the TPT2 free energy of the branched molecule system and $\Delta f_{q s u}^{T P T 2}$ is defined through Eq. (12). Further, we can employ Eq. (10) to simplify Eq. (12) as

$$
f_{m}^{T P T 2}=f_{m / l i n}^{T P T 2}+\Delta f_{\text {Branch }}^{T P T 2} .
$$

Here, $f_{m / l i n}^{T P T 2}$ is the TPT2 free energy for the linear isomer of length $m$ (Eq. (9)) and $\Delta f_{B \text { ranch }}^{T P T 2}$ is the difference in free energy between branched and linear isomers given by

$$
\Delta f_{\text {Branch }}^{T P T 2}=\Delta f_{q s u}^{T P T 2}-2 \Delta f^{T P T 2},
$$

where $\Delta f^{T P T 2}$ is given by Eq. (11). Since TPT2 only accounts for triplet interactions, the only way two spheres on different branches can interact with each other through the chain is if both branches extend from the same articulation segment (and they are the first sphere on the branch). For this reason, the difference in TPT2 free energy between a system of chains with two branches, with different articulation segments, and a system of chains with one branch should also be given by $\triangle f_{B r a n c h}^{T P T 2}$. When both branches are located on the same articulation segment $\Delta f_{\text {Branch }}^{T P T 2}$ should still represent the free energy difference between systems to a good approximation; we will assume from this point forward that $\Delta f_{B r a n c h}^{T P T 2}$ does not depend on the number of branches at a given articulation segment. We can then continue adding branches and obtain the TPT 2 correction to the free energy for an arbitrary branched molecule composed of $m$ segments and $N_{B}$ branches

$$
f_{m}^{T P T 2}=f_{m / l i n}^{T P T 2}+N_{B} \Delta f_{B r a n c h}^{T P T 2} .
$$

Now we need to evaluate $\Delta f_{B r a n c h}^{T P T 2}$ using Eq. (14). To do this, we consider the general branched chain solution ${ }^{15}$ which generalizes the results of Phan et al. ${ }^{14}$

$$
f_{m}^{T P T 2}=-\ln \sum_{n=0}^{N_{\max }} C_{n}^{m} \lambda^{n},
$$

where $C_{n}^{m}$ is the number of ways to partition a molecule of length $m$ into $n$ sections of three adjacent segments where two sections share at most one monomer, and $N_{\max }$ is the maximum number of three segment partitions allowed by the molecule; here, $C_{o}^{m}=1$. In Eq. (16), $\lambda$ is given by Eq. (6) for fixed bond angles and Eq. (8) for flexible molecules. As an example, a simple 5 segment star molecule is considered in Fig. 3; for this case, the coefficients are $C_{1}^{5}=6, C_{2}^{5}=3$, and $C_{n>2}^{5}=0$. Now we will consider the low density limit of Eq. (16)

$$
\left.f_{m}^{T P T 2}\right|_{\rho \rightarrow 0} \approx-\ln \left(1+C_{1}^{m} \lambda\right) \approx-C_{1}^{m} \lambda .
$$

For linear chains, we have $C_{1}^{m}=m-2$. In this limit, we can obtain the difference in free energy of the linear systems considered in Fig. 1 as

$$
\frac{\left.\Delta f^{T P T 2}\right|_{\rho \rightarrow 0}}{\lambda} \approx-(q+s-3)+(q-2)+(s-2)=-1 .
$$

Equation (18) is clearly independent of chain length and can also be derived through a Taylor series expansion of Eq. (11). Now we can evaluate $f_{m}^{T P T 2}$ in Eq. (12) in the low density limit for the systems given in Fig. 2 as

$$
\frac{\left.f_{m}^{T P T 2}\right|_{\rho \rightarrow 0}}{\lambda}=3-q-s-u
$$

which when combined with (12), (14), and (18) gives

$$
\frac{\left.\Delta f_{\text {Branch }}^{T P T 2}\right|_{\rho \rightarrow 0}}{\lambda}=\frac{\left.\Delta f^{T P T 2}\right|_{\rho \rightarrow 0}}{\lambda}=-1 .
$$

From Eq. (20), we see that in the low density limit the difference in free energy between branched and linear isomers is identical to the difference for linear chains discussed in Fig. 1. As density is increased higher order combinatorial coefficients $C_{k}^{m}(k>1)$ would have to be determined. Unfortunately, it would be extremely difficult to determine these coefficients in a general way, so we will assume the equality given by Eq. (20) holds at all densities

$$
\triangle f_{\text {Branch }}^{T P T 2} \approx \Delta f^{T P T 2} .
$$

This assumption will be justified by the accuracy of the resulting equation of state. Combining Eqs. (9), (11), (15), and

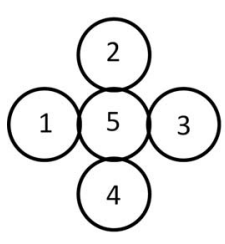

\begin{tabular}{c|c} 
Partitions $(\boldsymbol{n}=1)$ & Partitions $(\boldsymbol{n}=2)$ \\
\hline$(1,5,4)$ & $(1,5,4):(2,5,3)$ \\
$(1,5,2)$ & $(1,5,2):(3,5,4)$ \\
$(1,5,3)$ & $(1,5,3):(2,5,4)$ \\
$(2,5,4)$ & \\
$(2,5,3)$ & \\
$(3,5,4)$ & \\
\hline $\mathrm{C}_{1}=6$ & $\mathrm{C}_{2}=3$
\end{tabular}

FIG. 3. Triplet partitions of simple 4 arm star polymer. 
(21) we obtain our general branched TPT2 equation of state

$$
\begin{aligned}
f_{m}^{T P T 2}= & \left(N_{B}-1\right) \ln \left(\frac{1}{\sqrt{1+4 \lambda}}\right) \\
& +\left(N_{B}-m\right) \ln \left(\frac{1+\sqrt{1+4 \lambda}}{2}\right) .
\end{aligned}
$$

As will be shown shortly, this simple expression yields nearly identical results to the numerical TPT2 solution of Phan et al. ${ }^{14}$ for star molecules.

The free energy given by Eq. (15) should be applicable to other equations of state which provides a correction beyond first order. For instance, the TPT1-D equation of state ${ }^{16}$ creates chains by considering a dimer reference system. The theory was shown to give improved results over the typical TPT1-M treatment. In TPT1-D, the excess free energy for a system of linear chains of length $m$ is given as ${ }^{16}$

$$
f_{m}^{E}=f_{m}^{T P T 1-M}+f_{m}^{T P T 1-D},
$$

where

$$
f_{m}^{T P T 1-D}=-\left(\frac{m}{2}-1\right) \ln \left(\frac{g_{H D}(\sigma)}{g_{H S}(\sigma)}\right) .
$$

The term $g_{H D}(\sigma)$ is the site/site correlation function for a pair of hard dispheres. The difference in TPT1-D contributions to the free energy between a system of chains of length $q+s-1$ and the sum of systems of lengths $q$ and $s$ each at a packing fraction $\eta$ is given as

$$
\begin{aligned}
\Delta f^{T P T 1-D} & =f_{q+s-1}^{T P T 1-D}-f_{q}^{T P T 1-D}-f_{s}^{T P T 1-D} \\
& =-\frac{1}{2} \ln \left(\frac{g_{H D}(\sigma)}{g_{H S}(\sigma)}\right) .
\end{aligned}
$$

Since $\Delta f^{T P T 1-D}$ is independent of lengths $q$ and $s$, we can employ Eq. (15) to write the TPT1-D contribution for branched chains of length $m$

$$
f_{m}^{T P T 1-D}=-\left(\frac{m}{2}-1+\frac{N_{B}}{2}\right) \ln \left(\frac{g_{H D}(\sigma)}{g_{H S}(\sigma)}\right) .
$$

For $g_{H D}(\sigma)$, we use the correlation of Ghonasgi and Chapman ${ }^{16}$

$$
g_{H D}(\sigma)=\frac{1+2 \eta+26.45031 \eta^{6.17}}{2(1-\eta)^{2}} .
$$

The second virial coefficient $B_{2}$ is obtained as

$$
\begin{aligned}
B_{2} & =\left(\frac{\partial Z_{m}}{\partial \rho_{m}}\right)_{\rho_{m} \rightarrow 0} \\
& =m^{2}\left(\frac{1}{4}+\frac{5}{12 m}-\Psi\left(\frac{N_{B}-2}{m}+1\right)\right) \pi \sigma^{3},
\end{aligned}
$$

where $\Psi$ is a constant which depends on the model

$$
\Psi=\left\{\begin{array}{lc}
0.03883 & \text { TPT2 } \\
0.125 & \text { TPT1 }- \text { D }
\end{array} .\right.
$$

From Eq. (28), it is clear that increasing the number of branches decreases $B_{2}$, this is in agreement with previous simulation results for other hard alkane models ${ }^{26}$ and represents a significant qualitative improvement over the linear form of the theories. Since $\Psi$ is larger for the TPT1-D model, TPT1D predicts a larger decrease (as compared to TPT1-M) in the second virial coefficient than TPT2.

To test the athermal branched TPT2 and TPT1-D equations of state, we compare theory predictions to the simulation results of Yethiraj and $\mathrm{Hall}^{27}$ for the compressibility factor of star molecules. The star molecules are composed of $a$ arms of length $b$ with a total segment number of $m=a \times b+1$; the results can be found in Table I. The predictions of TPT1-D and TPT2 for linear chains are included for comparison, as are the numerical TPT2 solution for star polymers of Phan et al. ${ }^{14}$ The most important conclusion to draw from Table I is the fact that the approximate analytical branched TPT2 solution given by Eq. (22) is nearly identical to the numerical TPT2 solution of Phan et al. ${ }^{14}$ validating our solution methodology. The general effect of including branching effects is to decrease the compressibility factor. The last row of Table I gives the average absolute deviation $A A D=$ average $\left(\left|Z_{m}^{\text {sim }}-Z_{m}^{\text {theo }}\right| / Z_{m}^{\text {sim }}\right)$ between theory and simulation. For each case, the branched form of the theories are more accurate than the linear forms, with branched TPT1$\mathrm{D}$ being the most accurate.

To test the theory further, we can compare theoretical predictions of $B_{2}$ to the simulations of $\mathrm{Lue}^{28}$ for the second virial coefficient of tri-functional dendrimers. Lue ${ }^{28}$ performed Monte Carlo simulations for dendrimers consisting of $m$ tangentially bonded hard spheres; simulations were performed for dendrimers up to generation 5. Figure 4 illustrates Lue's dendrimer model generations $0-2$. Figure 5 compares theoretical and simulation predictions for the second virial coefficient of dendrimers using the branched and linear solutions. For clarity, the theoretical results are presented as curves; however, they are actually discrete with a value for each generation of dendrimer. We have also included the Monte Carlo results of Yethiraj et al. ${ }^{29}$ for the second virial coefficient of linear chains. As can be seen, TPT1-D is much more accurate than TPT1-M or TPT2 for the second virial coefficient of linear chains; for $m<25$ TPT1-D is in excellent agreement with simulation. Like the linear chain case, TPT1$D$ yields more accurate predictions of $B_{2}$ for dendrimers than TPT2. The branched TPT1-D theory is in reasonable agreement with simulation for generations less than 3 , and loses accuracy for larger dendrimers.

\section{EXTENSION TO A LENNARD-JONES DIMER REFERENCE SYSTEM}

The branched TPT1-D version of the theory is easily extended to a $\mathrm{LJ}$ reference system by replacing the monomer and dimer correlation functions with that of a $\mathrm{LJ}$ fluid. In a $\mathrm{LJ}$ fluid, the monomers interact with the Lennard-Jones potential

$$
\phi_{L J}(r)=4 \varepsilon\left(\left(\frac{\sigma_{L J}}{r}\right)^{12}-\left(\frac{\sigma_{L J}}{r}\right)^{6}\right) .
$$

The quantity $\varepsilon$ is the depth of the potential minimum and $r=\sigma_{L J}$ is the distance that the potential vanishes. The excess free energy of a system of branched chains of $\mathrm{LJ}$ spheres where the spheres are bonded at $r=\sigma_{L J}$ is obtained through 
TABLE I. Comparison of compressibility factor $Z_{m}=\frac{P}{\rho_{m} k_{B} T}$ for star molecules consisting of $a$ arms of length $b$ to the Monte Carlo simulations of Yethiraj and Hall. $^{27}$

\begin{tabular}{|c|c|c|c|c|c|c|c|c|}
\hline Arm length & Number of arms & $\eta$ & MC $\left(\right.$ Yethiraj $\left.^{29}\right)$ & TPT2 (linear) & TPT2 (branch) $\left(\mathrm{Phan}^{14}\right)$ & TPT2 (branch) & TPT1-D (linear) & TPT1-D (branch) \\
\hline 5 & 3 & 0.1065 & $4.53 \pm 0.13$ & 5.17 & 5.15 & 5.15 & 4.74 & 4.62 \\
\hline 5 & 3 & 0.1983 & $12.38 \pm 0.26$ & 13.04 & 13.00 & 12.99 & 12.65 & 12.49 \\
\hline 5 & 3 & 0.2488 & $19.24 \pm 0.31$ & 20.27 & 20.23 & 20.22 & 19.98 & 19.82 \\
\hline 5 & 3 & 0.2911 & $27.31 \pm 0.80$ & 28.77 & 28.73 & 28.71 & 28.53 & 28.36 \\
\hline 5 & 3 & 0.3806 & $53.49 \pm 1.54$ & 58.57 & 58.52 & 58.49 & 58.00 & 57.74 \\
\hline 3 & 3 & 0.0571 & $1.92 \pm 0.02$ & 2.17 & 2.16 & 2.15 & 1.99 & 1.92 \\
\hline 3 & 3 & 0.1058 & $3.42 \pm 0.08$ & 3.71 & 3.69 & 3.69 & 3.46 & 3.35 \\
\hline 3 & 3 & 0.1527 & $5.43 \pm 0.14$ & 5.86 & 5.83 & 5.82 & 5.60 & 5.47 \\
\hline 3 & 3 & 0.1979 & $8.34 \pm 0.19$ & 8.76 & 8.73 & 8.72 & 8.54 & 8.39 \\
\hline 3 & 3 & 0.2434 & $12.29 \pm 0.34$ & 12.73 & 12.69 & 12.67 & 12.55 & 12.39 \\
\hline 3 & 3 & 0.2889 & $17.8 \pm 0.50$ & 18.48 & 18.44 & 18.42 & 18.35 & 18.17 \\
\hline 3 & 3 & 0.3356 & $24.81 \pm 0.69$ & 26.61 & 26.56 & 26.54 & 26.45 & 26.24 \\
\hline 3 & 3 & 0.3728 & $36.39 \pm 1.21$ & 35.45 & 35.41 & 35.38 & 35.18 & 34.92 \\
\hline 5 & 4 & 0.1067 & $4.96 \pm 0.09$ & 6.38 & 6.32 & 6.33 & 5.79 & 5.56 \\
\hline 5 & 4 & 0.2017 & $14.89 \pm 0.42$ & 17.10 & 17.01 & 17.02 & 16.58 & 16.27 \\
\hline 5 & 4 & 0.2932 & $35.85 \pm 0.87$ & 37.72 & 37.61 & 37.60 & 37.40 & 37.05 \\
\hline 5 & 4 & 0.3827 & $78.14 \pm 2.99$ & 77.28 & 77.15 & 77.12 & 76.48 & 75.93 \\
\hline 3 & 4 & 0.0563 & $2.1 \pm 0.08$ & 2.44 & 2.40 & 2.41 & 2.20 & 2.06 \\
\hline 3 & 4 & 0.1057 & $3.77 \pm 0.13$ & 4.42 & 4.36 & 4.37 & 4.08 & 3.85 \\
\hline 3 & 4 & 0.1533 & $6.31 \pm 0.13$ & 7.20 & 7.12 & 7.13 & 6.85 & 6.57 \\
\hline 3 & 4 & 0.2005 & $9.67 \pm 0.13$ & 11.13 & 11.04 & 11.04 & 10.83 & 10.52 \\
\hline 3 & 4 & 0.2443 & $15.04 \pm 0.34$ & 16.22 & 16.12 & 16.11 & 15.98 & 15.66 \\
\hline 3 & 4 & 0.2929 & $22.24 \pm 0.55$ & 24.12 & 24.02 & 24.01 & 23.95 & 23.60 \\
\hline 3 & 4 & 0.3751 & $41.35 \pm 0.48$ & 46.09 & 45.97 & 45.94 & 45.69 & 45.18 \\
\hline $\mathrm{AAD}$ & & & & $9.6 \%$ & $9.1 \%$ & $9.1 \%$ & $7.6 \%$ & $6.0 \%$ \\
\hline
\end{tabular}

a simple modification of the results of Sec. II as

$$
\begin{aligned}
f_{m}^{E}= & f_{m}^{m o n, L J}-(m-1) \ln g_{m o n, L J}\left(\sigma_{L J}\right) \\
& -\left(\frac{m}{2}-1+\frac{N_{B}}{2}\right) \ln \left(\frac{g_{\operatorname{dim}, L J}\left(\sigma_{L J}\right)}{g_{m o n, L J}\left(\sigma_{L J}\right)}\right),
\end{aligned}
$$

where $f_{m}^{m o n, L J}$ is the excess free energy of a system of $\mathrm{LJ}$ spheres; for this quantity, we use the equation of state of Nicolas et al..$^{30}$ with the parameter set due to Johnson. ${ }^{31}$ The term $g_{\text {mon, } L J}\left(\sigma_{L J}\right)$ is the pair correlation function for a LJ fluid evaluated at the distance $r=\sigma_{L J}$ and $g_{\operatorname{dim}, L J}\left(\sigma_{L J}\right)$ is the corresponding correlation function for a pair of $\mathrm{LJ}$ dimers where the dimers are composed of two spheres bonded at $r=\sigma_{L J}$. For the monomer and dimer LJ correlation functions, we use the results of Johnson et al. ${ }^{21,22}$

It should be noted that the current theory does not know the location or length of branches on the molecule. For instance, the theory will predict the same phase diagram for

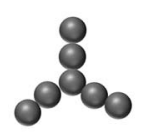

GEN 0

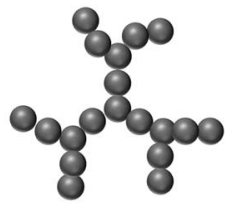

GEN 1

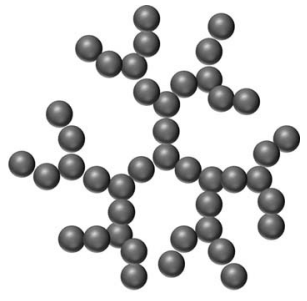

GEN 2
FIG. 4. Dendrimer model for generation $0 N_{B}=1$, generation $1 N_{B}=4$, and generation $2 N_{B}=10$. 2-methylpentane and 3-methylpentane where in reality the former has a lower critical temperature, ${ }^{32}$ the theory will also predict the same phase diagram for 3-ethylpentane and 3-methylhexane where it is known the former has a higher critical temperature. ${ }^{32}$

Direct comparison of the current theory to existing simulations for branched LJ molecules is difficult since all simulations are performed using fused spheres. ${ }^{33}$ To test the utility of Eq. (31), we will treat $m, \sigma_{L J}$, and $\varepsilon$ as parameters which are obtained by fitting Eq. (31) with $N_{B}=0$ to phase equilibria data (saturated liquid densities and pressures) for n-octane.

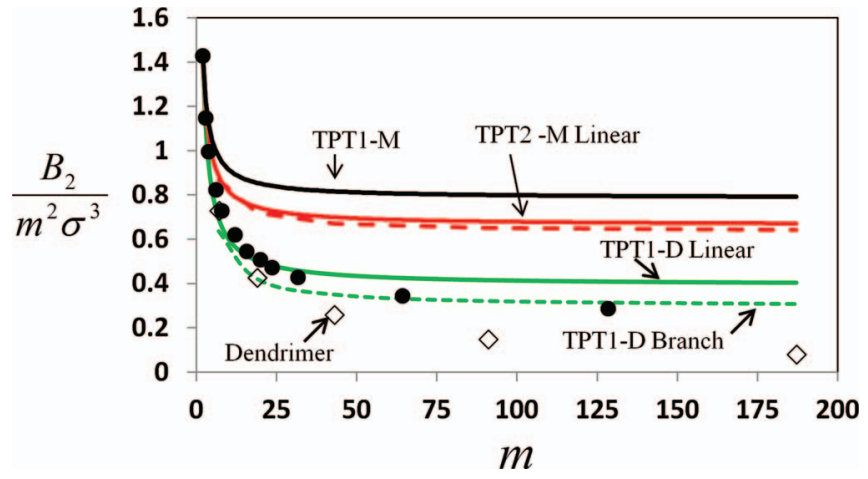

FIG. 5. Second virial coefficients for dendrimers and linear chains. Theoretical results are illustrated as lines for clarity; however, they are actually discrete with a value for each generation. Open diamonds give the simulation results of Lue ${ }^{28}$ for dendrimers and filled circles give the simulation results for linear chains of Yethiraj et al. ${ }^{29}$ The black line gives TPT1-M predictions, red lines give TPT2 predictions (solid - linear, dashed - branched), and green lines give TPT1-D predictions. 

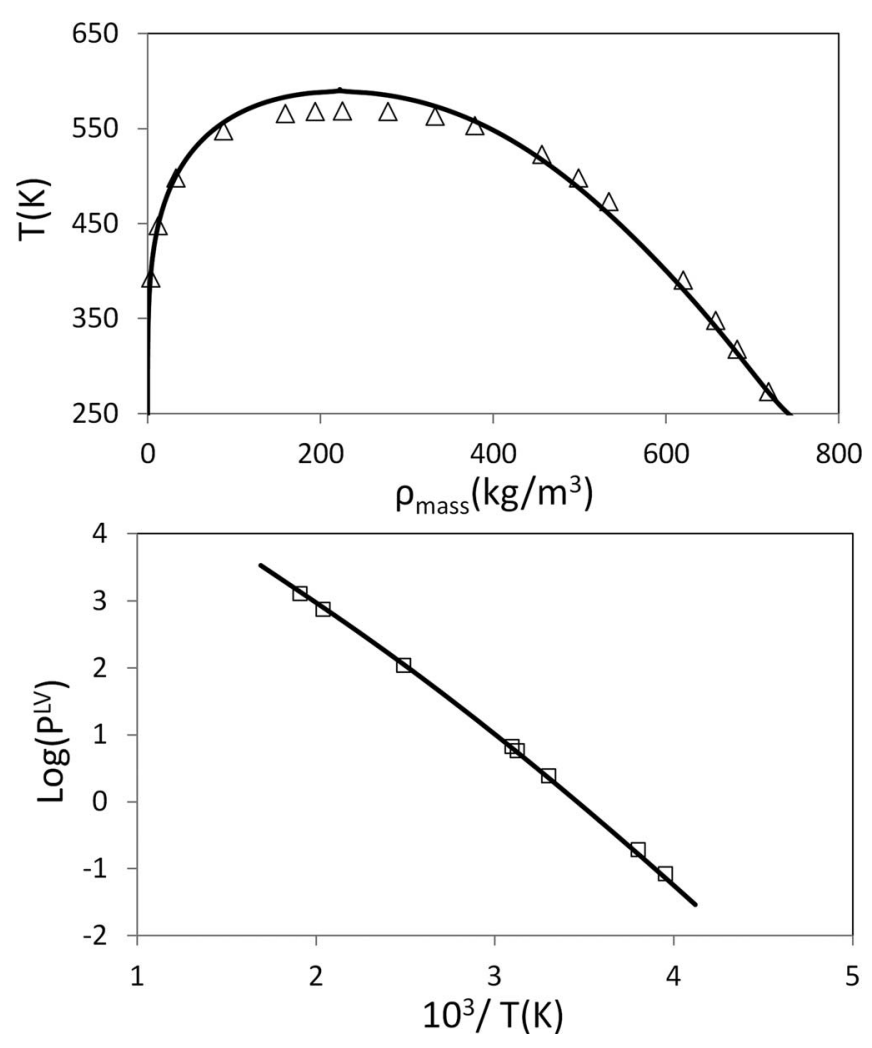

FIG. 6. Phase diagram (top) and vapor pressures in units of $\mathrm{kPa}$ (bottom) for $\mathrm{n}$-octane. Lines give theoretical predictions and symbols give experimental results. $^{34}$
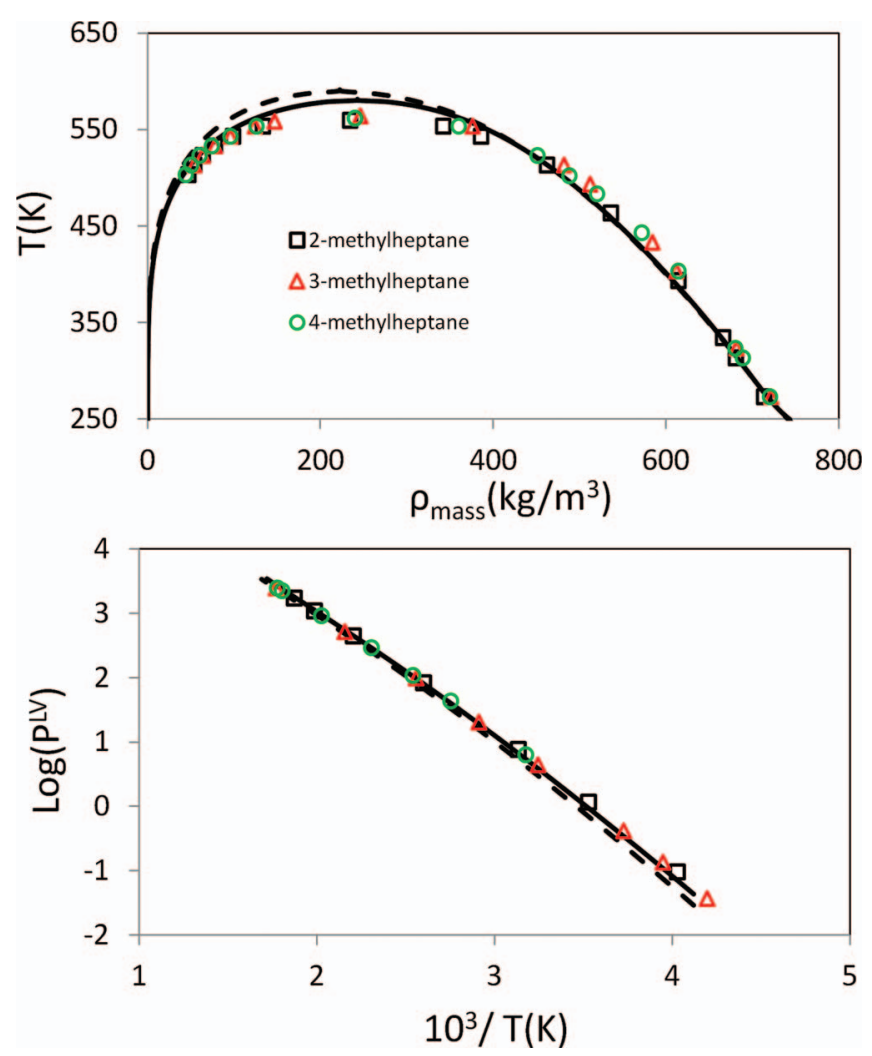

FIG. 7. Phase diagram (top) and vapor pressures in units of $\mathrm{kPa}$ (bottom) for 8 carbon isomers with a single methyl branch. Symbols give experimental results, ${ }^{34}$ dashed line is theoretical result for n-octane $N_{B}=0$ and solid lines give theoretical predictions for one branch molecules $N_{B}=1$.
Using this parameter set, we will explore the effect of branching on the phase diagram and vapor pressure $P^{L V}$ of a series of alkane isomers of formula $\mathrm{C}_{8} \mathrm{H}_{18}$. As a simple model, we assume that each pair of carbons (with hydrogens) is represented by a LJ segment, so for n-octane and all isomers we choose $m=4$. The remaining two parameters are then obtained by fitting Eq. (31) to saturated liquid density and vapor pressure data for n-octane, the results are $\sigma_{L J}=3.81 \AA$ and $\varepsilon / k_{B}=256.0 \mathrm{~K}$. Figure 6 compares the model results to experimental data for the T- $\rho$ phase diagram and the vapor pressure, as can be seen the theory is in good agreement experiment.

Now using this same parameter set, we can model the $\mathrm{C}_{8} \mathrm{H}_{18}$ isomers with a single methyl branch by setting $N_{B}=1$ in Eq. (31), these results can be seen in Fig. 7. The introduction of the branch results in a decrease in the critical temperature $T_{c}$, increase in the critical density $\rho_{c}$, and a general increase in vapor pressure; the vapor side of the phase diagram is affected more than the liquid side. The theory does a good job in predicting the change in properties which results from adding a branch. The theory does not know which carbon the branch is on; however, the experimental results also do not appear to strongly depend on the exact molecular form (the properties of 2-methylheptane are similar to 3-methylheptane).

Now to model $\mathrm{C}_{8} \mathrm{H}_{18}$ isomers with two branches, we simply set $N_{B}=2$ in Eq. (31), we are still using the n-octane parameter set. The comparison between theory and experiment can be found in Fig. 8. Like the 1 branch case, the addition of
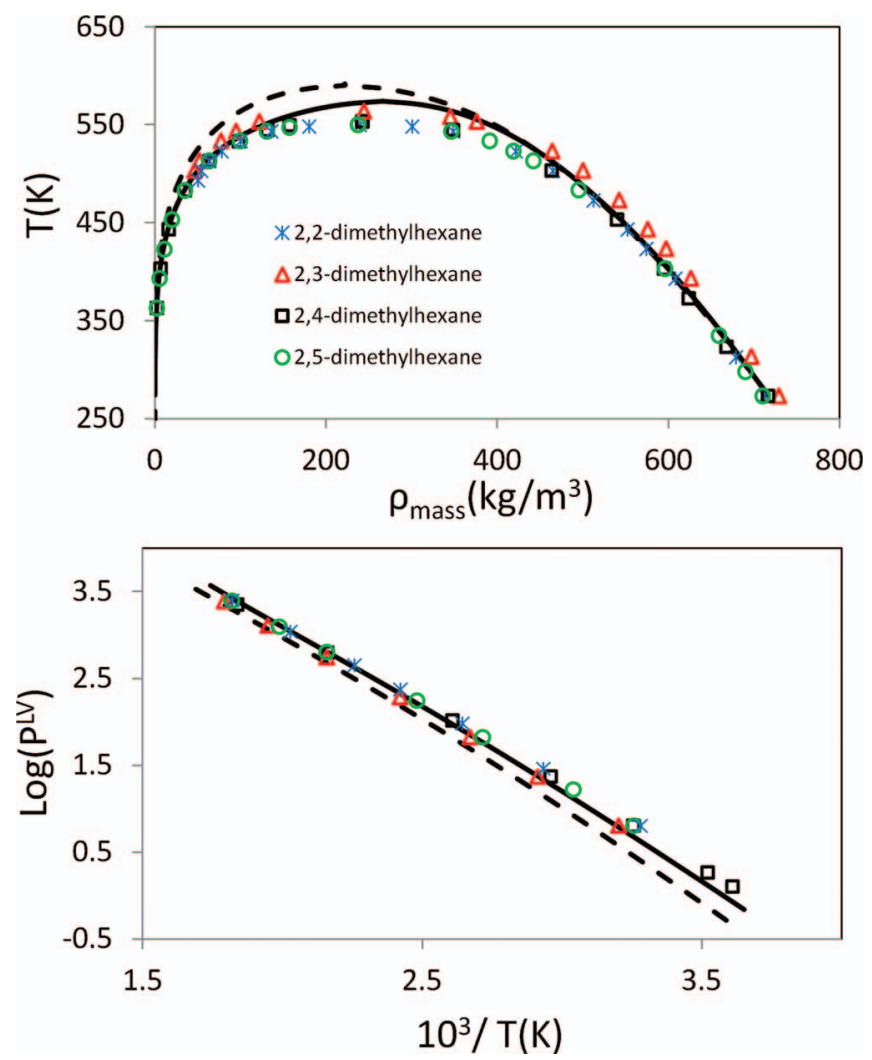

FIG. 8. Phase diagram (top) and vapor pressures in units of $\mathrm{kPa}$ (bottom) for 8 carbon isomers with two methyl branches. Symbols give experimental results, ${ }^{34}$ dashed line is theoretical result for n-octane $N_{B}=0$ and solid lines give theoretical predictions molecules with two branches $N_{B}=2$. 


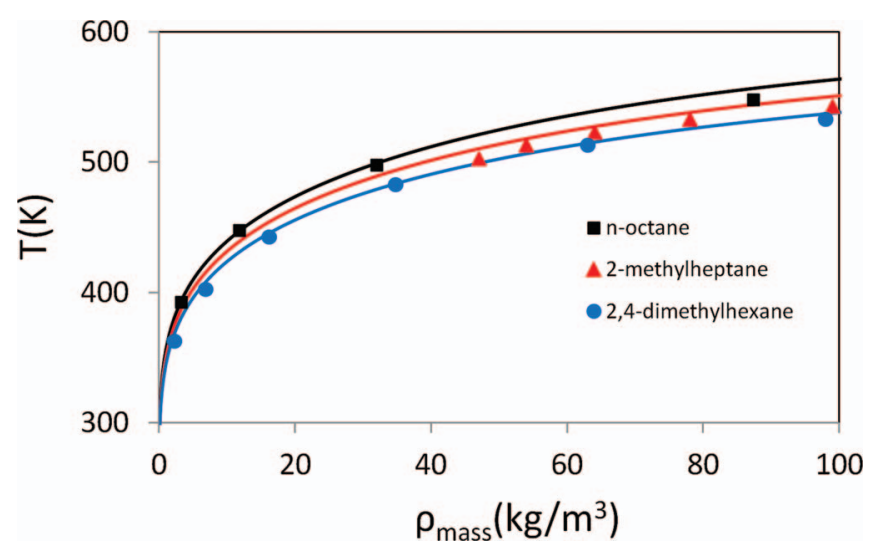

FIG. 9. Vapor side of phase diagram for n-octane, 2-methylheptane, and 2,4dimethyl hexane. Symbols give experimental results ${ }^{34}$ and curves give theoretical predictions.

two branches results in a decrease in the critical temperature $T_{c}$, increase in the critical density $\rho_{c}$, and a general increase in vapor pressure; however, now the effect is more pronounced than the 1 branch case. Experimentally, all shown isomers give nearly the same phase diagram with the exception of the isomer 2,3-dimethylhexane which shows a significant shift of the liquid side phase boundary to the right. Overall, the theory does a good job of predicting the change in properties due to branching.

Finally, Fig. 9 shows the vapor side of the phase boundary for n-octane, 2-methylheptane, and 2,4-dimethylhexane. This figure demonstrates the utility of the current approach; without any increase in complexity over the original theory for linear chains, ${ }^{22}$ we can accurately predict the effect of branching while using the parameter set for the linear isomer.

\section{CONCLUSIONS}

In the framework of Wertheim's perturbation theory, we have developed 2 new equations of state for branched hard chain fluids and an equation of state for branched LennardJones chains. Each equation of state is of no more complexity than their linear counterparts. One simply needs to identify the number of branches. It was found that theTPT1-D theory was more accurate than TPT2 for branched hard chain fluids in comparison to simulation data for the compressibility factor of star molecules and second virial coefficient of hard chain dendrimers.

Using the linear Lennard-Jones TPT1-D theory due to Johnson, ${ }^{22}$ we were able to extend the branched TPT1-D theory to a Lennard-Jones reference fluid which allows us to account for branching in Lennard-Jones chains. The resulting theory gives a three parameter equation of state which can be used to model real systems. We used the new theory to model a set of $\mathrm{C}_{8} \mathrm{H}_{18}$ isomers. The parameters were obtained by fitting the theory to the linear isomer n-octane and then the properties of the branched isomers were predicted with the new theory. The theory was shown to accurately predict the changes in the phase diagram and vapor pressure upon branching as compared to experimental data. The beauty of the new approach is that no additional complexity over the linear form of the theory is introduced.

\section{ACKNOWLEDGMENTS}

The financial support for this work was provided by the Robert A. Welch Foundation (Grant No. C-1241).

\section{APPENDIX: EVALUATION OF $\Delta f_{q s}^{T P T 2}$ FOR $q$ OR $s<3$}

In this Appendix, we show that the term $\triangle f_{q s}^{T P T 2}$ is independent of $q$ and $s$ for all $q, s \geq 2$. Equation (11) was developed using the slightly approximate free energy given by Eq. (9) which is only valid for $q, s \geq 3$ due the fact that $f_{2}^{T P T 2}$ must vanish. However, we show below that Eq. (10) still holds for $q, s=2$ if we allow for a hypothetical TPT2 correction for a dimer. Consider the case where both $q=2$ and $s=2$, then

$$
\begin{aligned}
f_{3}^{T P T 2}= & \Delta f_{22}^{T P T 2}=-\ln \left(\frac{1}{\sqrt{1+4 \lambda}}\right) \\
& -3 \ln \left(\frac{1+\sqrt{1+4 \lambda}}{2}\right) \\
= & -2 \ln \left(\frac{1}{\sqrt{1+4 \lambda}}\right)-4 \ln \left(\frac{1+\sqrt{1+4 \lambda}}{2}\right) \\
& +\Delta f^{T P T 2} \\
= & f_{2}^{T P T 2}+f_{2}^{T P T 2}+\Delta f^{T P T 2} .
\end{aligned}
$$

Here, $f_{2}^{T P T 2}$ is a hypothetical second order correction to the free energy for a dimer using the approximate free energy Eq. (9) with $q=2$. The last case to consider is for $q=2$ and $s \geq 3$

$$
\begin{aligned}
f_{2+s-1}^{T P T 2}= & f_{s}^{T P T 2}+\Delta f_{2, s}^{T P T 2} \\
= & -\ln \left(\frac{1}{\sqrt{1+4 \lambda}}\right)-(2+s-1) \ln \left(\frac{1+\sqrt{1+4 \lambda}}{2}\right) \\
= & -2 \ln \left(\frac{1}{\sqrt{1+4 \lambda}}\right)-(2+s) \ln \left(\frac{1+\sqrt{1+4 \lambda}}{2}\right) \\
& +\Delta f^{T P T 2} \\
= & f_{2}^{T P T 2}+f_{S}^{T P T 2}+\Delta f^{T P T 2} .
\end{aligned}
$$

Again $f_{2}^{T P T 2}$ is a hypothetical TPT2 correction for a dimer.

${ }^{1}$ M. Randic, J. Am. Chem. Soc. 97(23), 6609-6615 (1975).

${ }^{2}$ T. W. De Loos, W. Poot, and R. Lichtenthaler, J. Supercrit. Fluids 8(4), 282-286 (1995).

${ }^{3}$ W. Chapman, K. Gubbins, G. Jackson, and M. Radosz, Fluid Phase Equilib. 52, 31-38 (1989).

${ }^{4}$ M. Wertheim, J. Stat. Phys. 35(1), 19-34 (1984).

${ }^{5}$ M. Wertheim, J. Stat. Phys. 35(1), 35-47 (1984).

${ }^{6}$ M. Wertheim, J. Stat. Phys. 42(3), 459-476 (1986).

${ }^{7}$ M. Wertheim, J. Chem. Phys. 87, 7323 (1987).

${ }^{8}$ A. Galindo, P. J. Whitehead, G. Jackson, and A. N. Burgess, J. Phys. Chem. 100(16), 6781-6792 (1996).

${ }^{9}$ A. Gilvillegas, A. Galindo, P. J. Whitehead, S. J. Mills, G. Jackson, and A. N. Burgess, J. Chem. Phys. 106(10), 4168-4186 (1997).

${ }^{10}$ J. Gross and G. Sadowski, Ind. Eng. Chem. Res. 40(4), 1244-1260 (2001).

${ }^{11}$ W. G. Chapman, G. Jackson, and K. E. Gubbins, Mol. Phys. 65(5), 10571079 (1988)

${ }^{12}$ G. Jackson, W. G. Chapman, and K. E. Gubbins, Mol. Phys. 65(1), 1-31 (1988). 
${ }^{13}$ W. G. Chapman, Ph.D. dissertation, Cornell University, Ithaca, NY, 1988.

${ }^{14}$ S. Phan, E. Kierlik, M. Rosinberg, H. Yu, and G. Stell, J. Chem. Phys. 99, 5326 (1993)

${ }^{15}$ B. Marshall and W. G. Chapman, J. Phys. Chem. B 115(50), 15036-15047 (2011).

${ }^{16}$ D. Ghonasgi and W. G. Chapman, J. Chem. Phys. 100, 6633 (1994).

${ }^{17}$ J. Chang and S. I. Sandler, Chem. Eng. Sci. 49(17), 2777-2791 (1994).

${ }^{18}$ K. P. Shukla and W. G. Chapman, Mol. Phys. 93(2), 287-293 (1998).

${ }^{19}$ W. G. Chapman, J. Chem. Phys. 93, 4299 (1990).

${ }^{20}$ D. Ghonasgi and W. G. Chapman, AIChE J. 40(5), 878-887 (1994).

${ }^{21}$ J. K. Johnson, E. A. Mueller, and K. E. Gubbins, J. Phys. Chem. 98(25), 6413-6419 (1994).

${ }^{22}$ J. K. Johnson, J. Chem. Phys. 104, 1729 (1996).

${ }^{23}$ F. J. Blas and L. F. Vega, J. Chem. Phys. 115, 4355 (2001).

${ }^{24}$ F. J. Blas and L. F. Vega, J. Chem. Phys. 115, 3906 (2001).
${ }^{25}$ J. P. Hansen and I. R. McDonald, Theory of Simple Liquids (Academic Press, 2006).

${ }^{26}$ L. MacDowell and C. Vega, J. Chem. Phys. 109, 5670 (1998).

${ }^{27}$ A. Yethiraj and C. K. Hall, J. Chem. Phys. 94, 3943 (1991).

${ }^{28}$ L. Lue, Macromolecules 33(6), 2266-2272 (2000).

${ }^{29}$ A. Yethiraj, K. G. Honnell, and C. K. Hall, Macromolecules 25(15), 39793983 (1992).

${ }^{30}$ J. Nicolas, K. Gubbins, W. Streett, and D. Tildesley, Mol. Phys. 37(5), 1429-1454 (1979).

${ }^{31}$ J. K. Johnson, J. A. Zollweg, and K. E. Gubbins, Mol. Phys. 78(3), 591-618 (1993).

${ }^{32}$ T. E. Daubert, J. Chem. Eng. Data 41(3), 365-372 (1996).

${ }^{33}$ M. G. Martin and J. I. Siepmann, J. Phys. Chem. B 103(21), 4508-4517 (1999).

${ }^{34}$ TRC Thermodynamics Tables-Hydrocarbons, Thermodynamics Research Center, Texas A\&M University, College Station, TX. 